\title{
Enrichment of Oxygen-containing Low Concentration Coalbed Methane with CMS-3KT as the Adsorbent
}

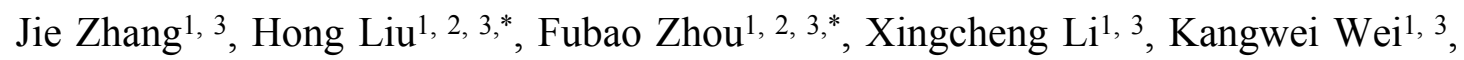
Jiaojiao Song ${ }^{1,3}$

1 Jiangsu Key Laboratory of Fire Safety in Urban Underground Space, Xuzhou 221116, PR China 2 National Engineering Research Centre for Coal Gas Control, Xuzhou 221116, PR China

3 Faculty of Safety Engineering, China University of Mining and Technology (CUMT), Xuzhou 221116, PR China

*Correspondence: liuhong2013@cumt.edu.cn; f.zhou@cumt.edu.cn. 


\section{Supporting information}
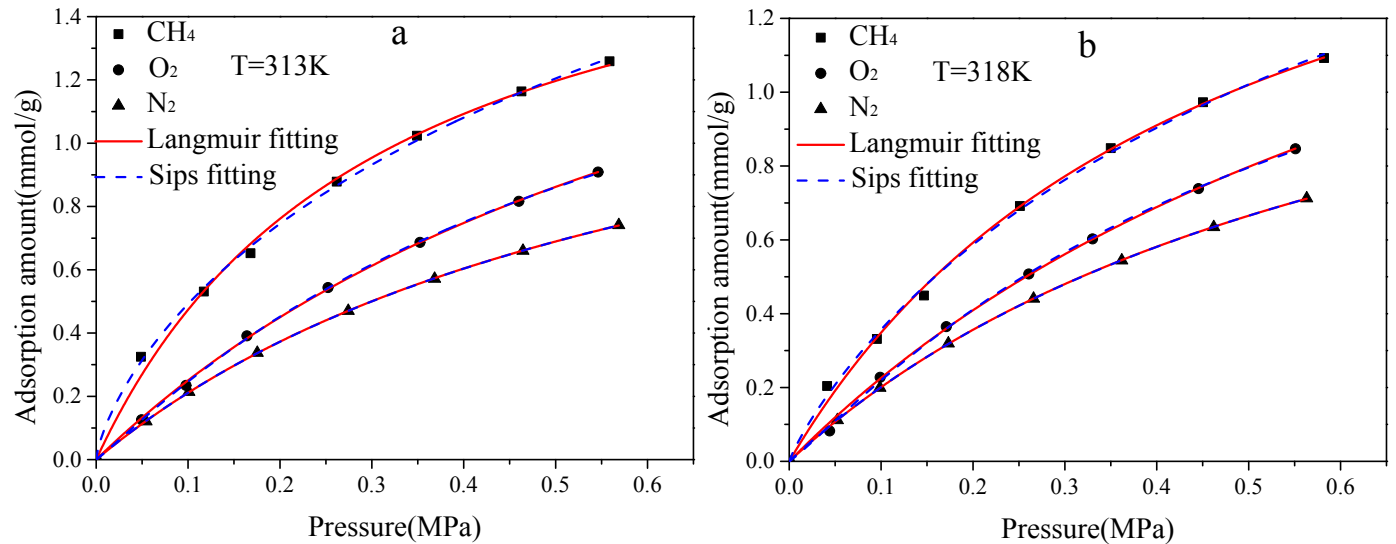

Figure S1. Adsorption isotherms of $\mathrm{CH}_{4}, \mathrm{O}_{2}$ and $\mathrm{N}_{2}$ in $313 \mathrm{~K}$ (a) and $318 \mathrm{~K}$ (b), respectively. 
Table S1. Adsorption amounts for $\mathrm{CH}_{4}, \mathrm{~N}_{2}$ and $\mathrm{O}_{2}$ on CMS-3KT in referenced studies.

\begin{tabular}{|c|c|c|c|c|c|c|c|}
\hline Adsorbent & Company & $\mathrm{T}(\mathrm{K})$ & $\mathrm{P}(\mathrm{MPa})$ & $\begin{array}{c}\mathrm{n}_{\mathrm{CH} 4} \\
(\mathrm{mmol} / \mathrm{g})\end{array}$ & $\begin{array}{c}\mathrm{n}_{\mathrm{N} 2} \\
(\mathrm{mmol} / \mathrm{g})\end{array}$ & $\begin{array}{c}\mathrm{n}_{\mathrm{O} 2} \\
(\mathrm{mmol} / \mathrm{g})\end{array}$ & Reference \\
\hline CMS-T3A & $\begin{array}{c}\text { Takeda } \\
\text { Chemical }\end{array}$ & 303 & $0-1.5$ & $<2.3$ & $<1.7$ & $<1.8$ & $\begin{array}{c}\text { (Bae et al., } \\
2004)\end{array}$ \\
\hline CMS-T3A & $\begin{array}{l}\text { Takeda } \\
\text { chemical }\end{array}$ & 293-313 & $0-1.6$ & $2.0-2.8$ & $1.4-2.0$ & $1.7-2.1$ & $\begin{array}{c}\text { (Bae and } \\
\text { Lee, 2005) }\end{array}$ \\
\hline $\begin{array}{c}\text { Takeda } \\
3 \mathrm{KT}\end{array}$ & $\begin{array}{c}\text { Changxin } \\
\text { g Shanli } \\
\text { Chemical } \\
\text { Material } \\
\text { Technolo }\end{array}$ & $298-343$ & $0-0.6$ & $1.1-1.8$ & $0.4-0.5$ & $0.5-0.7$ & $\begin{array}{l}\text { (Yang et } \\
\text { al., 2019) }\end{array}$ \\
\hline CMS & $\begin{array}{c}\text { gy } \\
\text { KURAR } \\
\text { AY } \\
\text { CHEMIC } \\
\text { AL }\end{array}$ & $293-323$ & $0-1.0$ & - & $1-1.5$ & $1.1-1.6$ & $\begin{array}{c}\text { (Park et } \\
\text { al., 2019a) }\end{array}$ \\
\hline $\begin{array}{c}\text { Takeda } \\
\text { CMS 3K }\end{array}$ & Takeda & $298-323$ & $0-1.6$ & $1.3-2.0$ & $0.7-1.0$ & - & $\begin{array}{c}\text { (Cavenati } \\
\text { et al., } \\
\text { 2005) }\end{array}$ \\
\hline CMS & $\begin{array}{c}\text { KURAR } \\
\text { AY } \\
\text { CHEMIC } \\
\text { AL } \\
\text { Air }\end{array}$ & $298-318$ & $0-1$ & - & $1-1.4$ & - & $\begin{array}{c}\text { (Park et } \\
\text { al., 2019b) }\end{array}$ \\
\hline CMS & $\begin{array}{l}\text { Products } \\
\text { and } \\
\text { Chemical } \\
\text { s }\end{array}$ & 293 & $0-0.1$ & - & $<0.3$ & $<0.3$ & $\begin{array}{c}\text { (O'koye et } \\
\text { al., 1997) }\end{array}$ \\
\hline CMS & $\begin{array}{l}\text { SHANLI } \\
\text { Chemical } \\
\text { Materials }\end{array}$ & $303-343$ & $0-0.73$ & $1.25-1.95$ & $0.65-1.0$ & - & $\begin{array}{l}\text { (Yang et } \\
\text { al., 2014) }\end{array}$ \\
\hline CMS-200 & $\begin{array}{c}\text { Yancheng } \\
\text { Jiutian }\end{array}$ & 293 & $0-0.53$ & $<1.4$ & $<0.85$ & - & $\begin{array}{l}\text { (Yang et } \\
\text { al., 2016) }\end{array}$ \\
\hline
\end{tabular}



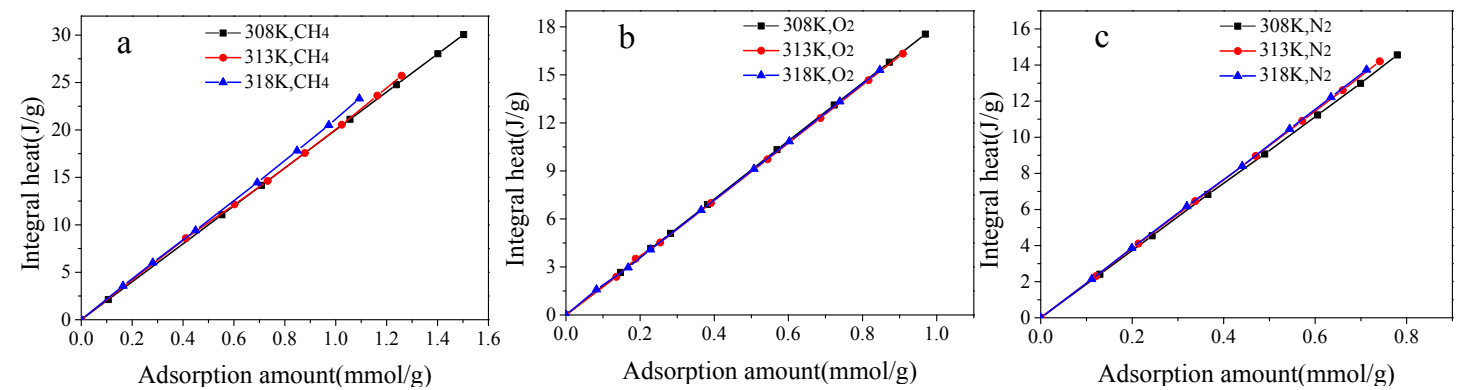

Figure S2. Integral heats of adsorption for $\mathrm{CH}_{4}(\mathrm{a}), \mathrm{O}_{2}$ (b) and $\mathrm{N}_{2}$ (c) in 308,313 and $318 \mathrm{~K}$, respectively. 

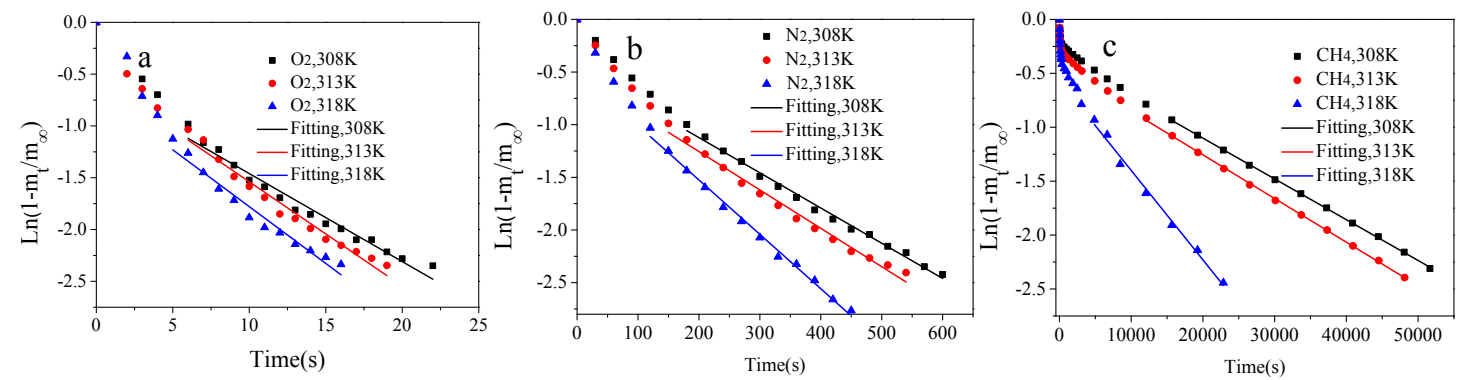

Figure S3. Linear fitting of adsorption uptake curves for $\mathrm{O}_{2}(\mathrm{a}), \mathrm{N}_{2}$ (b) and $\mathrm{CH}_{4}$ (c) on CMS-3KT at $0.1 \mathrm{MPa}$ in 308,313 and $318 \mathrm{~K}$, respectively. 